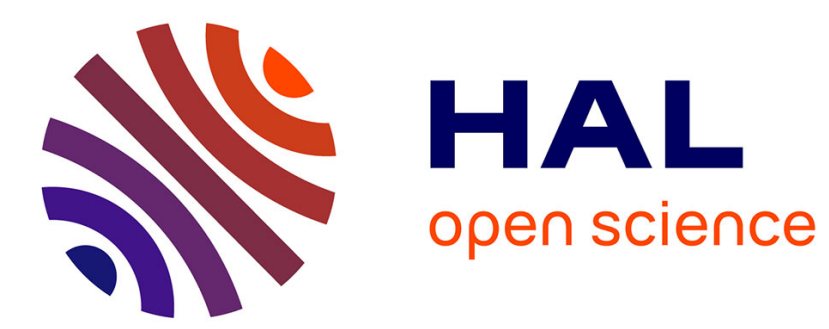

\title{
La détection cérébrale précoce des émotions
}

\author{
N. George
}

\section{To cite this version:}

N. George. La détection cérébrale précoce des émotions. Annales Médico-Psychologiques, Revue Psychiatrique, 2011, 169 (2), pp.112. 10.1016/j.amp.2010.12.003 . hal-00727593

\section{HAL Id: hal-00727593 \\ https://hal.science/hal-00727593}

Submitted on 4 Sep 2012

HAL is a multi-disciplinary open access archive for the deposit and dissemination of scientific research documents, whether they are published or not. The documents may come from teaching and research institutions in France or abroad, or from public or private research centers.
L'archive ouverte pluridisciplinaire HAL, est destinée au dépôt et à la diffusion de documents scientifiques de niveau recherche, publiés ou non, émanant des établissements d'enseignement et de recherche français ou étrangers, des laboratoires publics ou privés. 


\section{Accepted Manuscript}

Title: La détection cérébrale précoce des émotions

Author: N. George

PII: $\quad$ S0003-4487(10)00409-9

DOI: $\quad$ doi:10.1016/j.amp.2010.12.003

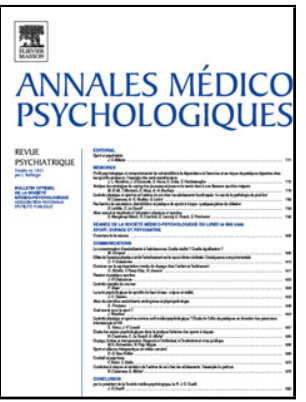

Reference: $\quad$ AMEPSY 1281

To appear in: $\quad$ Annales Médico-Psychologiques

Please cite this article as: George N, La détection cérébrale précoce des émotions, Annales medio-psychologiques (2010), doi:10.1016/j.amp.2010.12.003

This is a PDF file of an unedited manuscript that has been accepted for publication. As a service to our customers we are providing this early version of the manuscript. The manuscript will undergo copyediting, typesetting, and review of the resulting proof before it is published in its final form. Please note that during the production process errors may be discovered which could affect the content, and all legal disclaimers that apply to the journal pertain. 


\title{
Communication
}

La détection cérébrale précoce des émotions

The early detection of emotion by the human brain

N. George

CRICM, UMR 7225/UMR-S 975, UPMC/CNRS/INSERM, Équipe Cogimage (ex-LENA, UPR640) Bat. Paul Castaigne, $\sigma^{e}$ étage, Hôpital de la Salpêtrière,

47, Boulevard de l'Hôpital, 75651 Paris Cedex 13, France

Auteur correspondant: N. George, CRICM, UMR 7225/UMR-S 975, UPMC/CNRS/INSERM, Équipe Cogimage (ex-LENA, UPR640) Bat. Paul Castaigne, 6 étage, Hôpital de la Salpêtrière, 47, Boulevard de l'Hôpital, 75651 Paris Cedex 13, France Tél. : 0142161169

Fax : 0145862537

Adresse email : nathalie.george @upmc.fr

\section{Résumé}

$\mathrm{Au}$ sens littéral, les émotions sont ce qui nous fait sortir de nous. Elles ont fondamentalement un versant somatique, en tant que réponses comportementales à des stimuli externes ou internes, et un versant psychique, en tant qu'expériences subjectives, ressenties par le sujet. La perception émotionnelle, c'est-à-dire la détection et l'évaluation du caractère émotionnel de stimuli, est caractérisée par des phénomènes de capture attentionnelle et d'amplification perceptive, qui peuvent aboutir à une détection consciente et un traitement accru des stimuli émotionnels. Les modèles actuels proposent que l'amygdale jouerait un rôle central dans la détection cérébrale précoce des émotions. Cet article présente les preuves en faveur de tels modèles, en développant particulièrement les études récentes relatives aux modulations émotionnelles des réponses cérébrales précoces aux stimuli visuels (C1 et P1) observées en électro et magnéto-encéphalographie (EEG-MEG).

Mots clés : Amygdale ; EEG ; Émotion ; MEG ; Perception

\begin{abstract}
Emotions comprise a somatic and a psychological dimension. Indeed, they are both behavioural responses to internal or external stimuli and subjective experiences or feelings.
\end{abstract}


Emotional perception can be defined as the detection and the appraisal of the emotional value of encountered stimuli. Attention capture and perceptual amplification are two important properties of emotional perception, which can result in enhanced conscious perception and increased processing of emotional stimuli. It has been proposed that amygdala plays a central role in the early detection of emotional stimuli. This paper presents the evidence accumulated in support of this proposal. In particular, recent studies showing emotional modulation of early brain responses ( $\mathrm{C} 1$ and P1) to visual stimuli with electro- and magnetoencephalography (EEG and MEG) are reviewed.

Keywords: Amygdala; EEG; Emotion; MEG; Perception

\section{Les émotions}

$\mathrm{Au}$ sens littéral, les émotions sont ce qui nous fait sortir de nous. Elles sont communément définies comme des perturbations de l'état de l'organisme, généralement brusques et intenses, en réaction à un événement externe ou interne. Depuis Darwin [8], l'étude neuroscientifique des émotions insiste généralement sur le caractère primitif des émotions, soulignant les propriétés communes des réactions et de l'expressivité émotionnelle chez l'homme et l'animal, en particulier les primates non humains. Ce caractère primitif des émotions est généralement associé à leur valeur adaptative. Les émotions sont fondamentalement des réponses de l'organisme permettant une reconfiguration rapide de ses priorités et/ou une mobilisation de ses ressources - physiques et mentales - face à un événement perturbateur. Elles déterminent en particulier des comportements d'approche ou d'évitement en réponse à ces événements.

Les émotions ont une double facette : somatique et psychique. En effet, comme nous venons de le voir, elles sont un ensemble de réactions comportementales, incluant des modifications du rythme respiratoire et cardiaque, de la vasomotricité, de la tension musculaire... réactions qui préparent l'organisme à l'action, mais elles sont aussi une expérience phénoménale subjective, un ressenti, dont le signe extérieur le plus visible est sans doute l'expression faciale. La question de savoir si c'est la composante somatique ou psychique qui est première dans les émotions fait toujours débat aujourd'hui. Cette double constitution met cependant en lumière la fonction de communication des émotions, sans doute particulièrement développée chez l'être humain. L'expression d'émotions, qu'elle soit vocale, 
corporelle ou faciale, permet de signaler une intention d'action; dans le domaine des émotions positives, elle indique par exemple un objet d'intérêt dans l'environnement, ou permet de renforcer des comportements positifs, prosociaux ou bénéfiques pour l'individu. Ainsi, les expressions faciales des émotions constituent des signaux non verbaux de communication essentiels dans les interactions interpersonnelles dont Darwin a écrit qu'ils étaient le premier moyen de communication entre la mère et son enfant [8].

\section{La perception émotionnelle}

Qu'entendre par les termes de «stimuli émotionnels» et de «perception émotionnelle»? Il s'agit de la détection et de l'évaluation par le cerveau de la valeur émotionnelle de stimuli perçus. Un stimulus ou un événement n'est pas émotionnel en tant que tel. Il l'est parce qu'il déclenche une émotion ou que l'organisme lui attribue une valeur émotionnelle. Certains stimuli ont une valeur émotionnelle quasi universelle, au sens où ils sont détectés comme émotionnels par tous les êtres humains, quelle que soit l'origine, innée ou acquise, de ce caractère émotionnel. On considère généralement que c'est le cas des expressions faciales des émotions de base, telle la peur en particulier. D'autres stimuli ont une valeur émotionnelle idiosyncrasique, liée à l'histoire d'un individu particulier, et émotionnelle uniquement pour lui. C'est bien sûr le plus souvent le premier type de stimuli qu'on qualifie d'émotionnel et qu'on utilise dans les études neuroscientifiques de la perception émotionnelle. Notons aussi que le développement de la connaissance des circuits cérébraux des émotions et des méthodes d'imagerie cérébrale fonctionnelle pour les étudier conduit souvent à qualifier de stimuli émotionnels les stimuli déclenchant l'activation des réseaux cérébraux de l'émotion.

Deux propriétés principales de la perception émotionnelle méritent d'être soulignées. Tout d'abord, il existe une facilitation du traitement perceptif des stimuli émotionnels. En d'autres termes, la détection cérébrale du caractère émotionnel d'un stimulus conduit à une facilitation et une amplification de son traitement. Cela a pu par exemple être mis en évidence dans des expériences de clignement attentionnel [4]. Dans ce type d'étude, on présente de façon sérielle et rapide une liste de mots écrits, la tâche du sujet étant de rapporter deux mots cibles, présentés en vert par exemple. Lorsque l'intervalle de temps entre le premier et le second mot cible est relativement court, la probabilité de rapporter le second mot diminue de façon importante. C'est le phénomène de clignement attentionnel. Anderson et Phelps [4] ont démontré que ce phénomène était diminué lorsque le second mot cible était un mot 
émotionnel. Cela démontre une facilitation de la détection consciente des stimuli émotionnels par rapport aux stimuli «neutres » (dénués de signification émotionnelle).

Par ailleurs, la détection de stimuli émotionnels peut se faire de façon non consciente. Ainsi, des visages exprimant la colère et conditionnés pour renforcer leur valeur aversive peuvent être présentés de façon subliminale, en les exposant pendant un temps très court (30 ms) et aussitôt suivis d'un visage neutre $(45 \mathrm{~ms})$ masquant. Seul le visage neutre est alors perçu consciemment par les sujets. Pourtant, le visage émotionnel non perçu engendre une réponse électrodermale accrue, témoin d'une réaction neurovégétative liée à l'éveil émotionnel. De plus, une activation de l'amygdale, région centrale dans l'évaluation des stimuli émotionnels, est observée en réponse aux visages émotionnels masqués [17].

Ces phénomènes liés à la perception de stimuli émotionnels reflètent la saillance de ces stimuli, hautement pertinents, pour l'organisme, et leur capacité à capturer l'attention à un niveau préconscient et conscient, aboutissant à une détectabilité accrue et une amplification du traitement perceptif. Le terme d'attention émotionnelle a été proposé pour décrire les mécanismes cérébraux sous-jacents [2,21].

\section{La détection cérébrale des émotions}

À la suite des travaux sur les émotions chez l'animal [14], l'amygdale est devenue la structure phare dans les études sur les circuits cérébraux de la perception émotionnelle chez l'homme. L'amygdale est une structure du lobe temporal médian située en avant de l'hippocampe, et d'origine phylogénétique ancienne. Elle est au carrefour de la détection et de l'évaluation des stimuli émotionnels, d'une part, et des réactions émotionnelles, d'autre part. L'amygdale reçoit des afférences des différents cortex sensoriels. Ainsi, dans le domaine visuel, la voie de traitement geniculo-striée-extrastriée des stimuli aboutit - entre autres dans l'amygdale, et l'amygdale envoie de nombreuses connexions en retour vers tous les niveaux de la voie visuelle, en particulier ventrale [3]. Ces connexions en retour constituent le substrat neuroanatomique de l'amplification perceptive des stimuli émotionnels. En outre, il a été proposé qu'il existe une voie de traitement sous-cortical susceptible de véhiculer très rapidement des informations visuelles relativement grossières jusqu'à l'amygdale. Cette voie impliquerait le colliculus supérieur, une structure du tectum du mésencéphale, et le pulvinar, un noyau du thalamus [15]. Cette voie sous-corticale ainsi qu'une portion rapide de la voie corticale seraient responsable de la détection cérébrale précoce des émotions [1]. 
Quelles sont les preuves à l'appui de ce modèle de la perception émotionnelle ? Les études dans ce domaine sont très nombreuses, et nous n'en retiendrons que quelques exemples. Dans la tâche de clignement attentionnel évoquée plus haut, il a été démontré qu'un patient souffrant d'une lésion bilatérale de l'amygdale ne présentait pas de réduction du clignement pour les mots émotionnels [4]. Chez ce patient, il n'y a pas de facilitation de la détection consciente des stimuli émotionnels par rapport aux stimuli neutres. De plus, dans les études d'imagerie fonctionnelle cérébrale par résonance magnétique nucléaire (IRMf), l'amplification du traitement perceptif de stimuli émotionnels tels que des visages effrayés, au niveau des régions extrastriées temporales ventrales, est dépendante de l'intégrité de l'amygdale [22]. Ces auteurs ont démontré que cette amplification n'était pas observée chez des patients souffrant de lésion de l'amygdale. En outre, l'activation de l'amygdale en réponse à des visages exprimant la peur covarie avec celle des régions sous-corticales du colliculus supérieur et du pulvinar [18].

\section{Une détection précoce?}

Les preuves relatives à une détection cérébrale précoce des émotions nécessitent néanmoins l'emploi de méthodes permettant de suivre avec précision la dynamique temporelle des réponses cérébrales à des stimuli émotionnels. Seules les méthodes d'imagerie cérébrale électrophysiologique, de type électroencéphalographie (EEG) et magnétoencéphalographie (MEG) offrent la possibilité de suivre milliseconde après milliseconde l'activité électrique neuronale telle qu'enregistrée à un niveau macroscopique (à la surface de la tête) chez le sujet sain. Les réponses évoquées par la présentation de différents types de stimuli et lors de la réalisation de différentes tâches cognitives ont été largement étudiées. Ainsi, la présentation de stimuli visuels induit une onde $\mathrm{C} 1$, culminant entre $50 \mathrm{~ms}$ et $90 \mathrm{~ms}$, qui traduit les étapes les plus précoces du traitement visuel, impliquant en particulier le cortex visuel primaire. Cette onde est suivie de l'onde P1, qui culmine vers $100 \mathrm{~ms}$, et met en jeu des régions visuelles extrastriées.

Dans le domaine de la perception de stimuli émotionnels, les études en EEG et en MEG ont pendant longtemps principalement rapporté des réponses cérébrales tardives aux émotions. Ces réponses, observées à partir de $250 \mathrm{~ms}$, voire $300 \mathrm{~ms}$ ou $400 \mathrm{~ms}$ après le début de la présentation d'un stimulus émotionnel, pourraient correspondre à des étapes d'évaluation consciente et d'extraction de la signification conceptuelle des stimuli 
émotionnels, ainsi qu'à divers processus cognitifs. Cependant, de plus en plus d'études accumulent les preuves en faveur de réponses cérébrales précoces aux émotions [11].

Ainsi, diverses études récentes ont démontré des influences émotionnelles dès les premières $50 \mathrm{~ms}$ à $90 \mathrm{~ms}$ du traitement visuel, c'est-à-dire dans la fenêtre temporelle de l'onde C1 du PE visuel [19,20]. Dans notre groupe, nous avons utilisé un paradigme de répétition incidente de visages effrayés, joyeux et neutres afin d'évaluer s'il existait un encodage différentié précoce de ces différents types de visages, dans une étude combinant des enregistrements MEG et EEG [16]. Nous avons ainsi pu montrer des modulations des effets de répétition des visages dès $40 \mathrm{~ms}$ à $50 \mathrm{~ms}$ après la présentation des stimuli, démontrant que l'expression émotionnelle peut influencer les étapes les plus précoces du traitement des visages. D'autres études ont montré des modulations émotionnelles de l'onde P1 du PE visuel, en réponse à des visages mais aussi à d'autres types de stimuli et incluant des modulations par une émotion positive (de joie) [6,9]. Ces influences émotionnelles pourraient être accrues chez les sujets présentant un niveau d'anxiété élevé [12]. En d'autres termes, la détection cérébrale précoce des émotions pourrait varier en fonction de traits de personnalité individuels. Soulignons que ces influences précoces des émotions sont antérieures aux réponses cérébrales typiquement associées à l'analyse perceptive des visages en tant que tels. Ainsi, la détection cérébrale des émotions semble pouvoir être plus précoce que les processus de catégorisation et de reconnaissance visuelle. Cette détection précoce pourrait être dans certains cas au moins basée sur des propriétés de relativement bas niveau des stimuli, qui seraient prédictives du caractère émotionnel des stimuli.

Nous nous intéressons depuis peu à l'étude directe de la dynamique temporelle des réponses de l'amygdale à partir des signaux MEG, autrement accessible uniquement à partir d'enregistrements intra-cérébraux effectués dans un contexte préchirurgical chez des patients épileptiques [13]. Nous utilisons pour cela des méthodes de segmentation des structures du lobe temporal médian à partir des images IRM anatomiques individuelles [7]. Cela permet de modéliser explicitement la contribution de sources placées dans l'amygdale au signal enregistré en MEG [5]. Nous avons pu ainsi étudier le décours temporel des réponses de l'amygdale à des visages effrayés et neutres. Ces réponses sont observées à partir de $80 \mathrm{~ms}$ environ, et elles sont plus amples pour les visages effrayés que pour les visages neutres entre $140 \mathrm{~ms}$ et $170 \mathrm{~ms}[10]$.

L'ensemble de ces études étayent l'hypothèse d'une détection cérébrale précoce des émotions impliquant en particulier l'amygdale. Bien que nous ayons ici seulement effleuré le sujet, il ressort aussi des études en EEG et MEG qu'il existe différentes étapes de traitement 
des stimuli émotionnels, distribuées dans le temps. Différentes structures cérébrales pourraient être impliquées dans ces différentes étapes de traitement, mais il est aussi possible qu'une même structure intervienne à différentes étapes, avec des rôles fonctionnels distincts [1]. Seules des méthodes d'imagerie cérébrale fonctionnelle sophistiquées, alliant résolution temporelle et pouvoir de localisation, permettront de caractériser complètement l'organisation spatio-temporelle du traitement cérébral des émotions. De telles études offrent d'intéressantes perspectives dans le cadre de l'approche clinique. De nombreuses pathologies neurologiques et psychiatriques impliquent des désordres de la perception émotionnelle. Il serait intéressant de tester quelles étapes du traitement des émotions sont susceptibles d'être affectées par ces différentes pathologies et par leur traitement.

\section{Conflit d'intérêt : à compléter par l'auteur}

\section{Références}

[1] Adolphs R. Neural systems for recognizing emotion. Curr Opin Neurobiol 2002;12:16977.

[2] Adolphs R, Spezio M. Role of the amygdala in processing visual social stimuli. Prog Brain Res 2006;156:363-78.

[3] Amaral DG, Behniea H, Kelly JL. Topographic organization of projections from the amygdala to the visual cortex in the macaque monkey. Neuroscience 2003;118:1099-120.

[4] Anderson AK, Phelps EA. Lesions of the human amygdala impair enhanced perception of emotionally salient events. Nature 2001;411:305-9.

[5] Attal Y, Bhattacharjee M, Yelnik J, Cottereau B, Lefevre J, Okada Y, Bardinet E, Chupin M, Baillet S. Modeling and detecting deep brain activity with MEG \& EEG. Conf Proc IEEE Eng Med Biol Soc 2007. p.4937-40.

[6] Carretie L, Hinojosa JA, Martin-Loeches M, Mercado F, Tapia M. Automatic attention to emotional stimuli: neural correlates. Hum Brain Mapp 2004;22:290-9.

[7] Chupin M, Hammers A, Bardinet E, Colliot O, Liu RS, Duncan JS, Garnero L, Lemieux L. Fully automatic segmentation of the hippocampus and the amygdala from MRI using hybrid prior knowledge. Med Image Comput Comput Assist Interv 2007;10:875-82.

[8] Darwin C. The expression of the emotions in man and animals. London: John Murray; 1872.

[9] Dubal S, Foucher A, Jouvent R, Nadel J. Human brain spots emotion in non humanoid robots. Soc Cogn Affect Neurosci; 2010. 
[10] Dumas T, Attal Y, Chupin M, Jouvent R, Dubal S, George N. MEG study of amygdala responses during the perception of emotional faces and gaze. In: Supek S, Sušac A (eds). 17th International Conference on Biomagnetism Advances in Biomagnetism - Biomag 2010 (IFMBE Proceedings), vol 28. Springer Berlin Heidelberg. p.330-3.

[11] George N. Perception and elicitation of emotion from faces. In: Vuilleumier P, Armony JL (eds). Handbook of human affective neuroscience. Cambridge University Press; 2010.

[12] Holmes A, Nielsen MK, Green S. Effects of anxiety on the processing of fearful and happy faces: an event-related potential study. Biol Psychol 2008;77:159-73.

[13] Krolak-Salmon P, Henaff MA, Vighetto A, Bertrand O, Mauguiere F. Early amygdala reaction to fear spreading in occipital, temporal, and frontal cortex: a depth electrode ERP study in human. Neuron 2004;42:665-76.

[14] LeDoux JE. The Emotional Brain. New-York: Simon \& Schuster; 1996.

[15] Linke R, De Lima AD, Schwegler H, Pape HC. Direct synaptic connections of axons from superior colliculus with identified thalamo-amygdaloid projection neurons in the rat: possible substrates of a subcortical visual pathway to the amygdala. J Comp Neurol 1999;403:158-70.

[16] Morel S, Ponz A, Mercier M, Vuilleumier P, George N. EEG-MEG evidence for early differential repetition effects for fearful, happy and neutral faces. Brain Res 2009;1254:84-98. [17] Morris JS, Ohman A, Dolan RJ. Conscious and unconscious emotional learning in the human amygdala. Nature 1998;393:467-70.

[18] Morris JS, Ohman A, Dolan RJ. A subcortical pathway to the right amygdala mediating "unseen" fear. Proc Natl Acad Sci USA 1999;96:1680-5.

[19] Pourtois G, Grandjean D, Sander D, Vuilleumier P. Electrophysiological correlates of rapid spatial orienting towards fearful faces. Cereb Cortex 2004;14:619-33.

[20] Stolarova M, Keil A, Moratti S. Modulation of the C1 visual event-related component by conditioned stimuli: evidence for sensory plasticity in early affective perception. Cereb Cortex 2006;16:876-87.

[21] Vuilleumier P. How brains beware: neural mechanisms of emotional attention. Trends Cogn Sci 2005;9:585-94.

[22] Vuilleumier P, Richardson MP, Armony JL, Driver J, Dolan RJ. Distant influences of amygdala lesion on visual cortical activation during emotional face processing. Nat Neurosci 2004;7:1271-8. 


\section{Discussion}

Dr J. Birnbaum - S'agit-il de réponses cérébrales aux stimuli internes ?

$\operatorname{Pr}$ M. Bourgeois - Félicitations pour ce programme et ces études qui légitiment notre spécialité dans sa dynamique et son futur au sein de la médecine. Anatomiquement, amygdale et hippocampe sont-ils nettement distincts comme le suggèrent vos images alors que souvent on a pu dire qu'ils étaient trop intriqués?

Pr J.-M. Vanelle - Beaucoup d'études sur les émotions semblent utiliser le paradigme des visages et leurs expressions de diverses émotions : peur, colère, joie...

Existe-t-il d'autres moyens d'études, faisant appel à d'autres organes des sens que la vue comme l'ouïe ou l'odorat?

$\operatorname{Pr}$ M. Laxenaire - Votre méthode permet-elle de résoudre le dilemme posé par William James que vous avez évoqué tout à l'heure. Est-ce l'émotion ou le processus cérébral qui est primitif?

Réponse du Rapporteur - au Dr Birnbaum - Les réponses émotionnelles peuvent être déclenchées par des stimuli internes ou externes. Les études que j'ai évoquées ont étudié les réponses cérébrales précoces à des stimuli externes ayant une valeur émotionnelle intrinsèque (comme les expressions faciales) ou acquise (par association avec un contexte émotionnel par exemple). La temporalité des réponses cérébrales aux stimuli internes est difficile à étudier, et inconnue à ce jour.

$\mathrm{Au}$ Pr M. Bourgeois - Amygdale et hippocampe sont anatomiquement distincts au sens où l'on peut, par des méthodes de segmentation anatomique automatique, isoler les surfaces respectives de ces deux structures. Ces régions sont néanmoins fortement interconnectées, et cela se traduit sur le plan fonctionnel par la richesse et la complexité des interactions entre mémoire et émotions.

Au Pr J.-M. Vanelle - Mes propres recherches m'ont conduit à biaiser ma présentation en donnant une place privilégiée aux visages. Au cours des dix ou vingt dernières années, ces derniers ont néanmoins été effectivement très utilisés dans le cadre de l'étude de la perception émotionnelle chez l'homme. Mais de nombreuses études s'intéressent aussi à la perception 
émotionnelle auditive, et des études moins nombreuses à la perception émotionnelle liée aux odeurs, ces dernières nécessitant des dispositifs techniques relativement complexes.

$\mathrm{Au} \operatorname{Pr}$ M. Laxenaire - Cette question reste difficile. La réponse que l'on peut y apporter est limitée par la dynamique temporelle intrinsèque des méthodes d'étude utilisées. Ainsi, les réponses «corporelles » neurovégétatives associées aux émotions que l'on peut enregistrer sous la forme de réponses électrodermales par exemple ont une dynamique temporelle lente, alors que les enregistrements EEG et MEG permettent de suivre les réponses cérébrales avec une dynamique extrêmement rapide. Ce que l'on peut dire, quand il s'agit de stimulus externes, c'est que la perception de ces stimulus met très rapidement en jeu un réseau de régions impliquées dans la perception émotionnelle, le ressenti et la réaction émotionnelle, avec des activations probablement au moins en partie voire massivement parallèles et des boucles de rétroaction importantes entre les différentes régions et entre les différents processus. Ainsi, si l'on définit l'émotion comme intégrant des composantes somatiques et cérébrales, la question de la primauté de l'une de ces composantes lors de la perception d'un stimulus émotionnel est rapidement obsolète à l'échelle du traitement cérébral. Il faut noter aussi que le poids de ces composantes est éventuellement variable, selon les situations, les stimuli, voire les individus. 